FERMILAB-Conf-89/72

\title{
A Magnetically Switched Kicker for Proton Extraction*
}

John Dinkel and James Biggs

Fermi National Accelerator Laboratory

P.O. Box 500, Batavia, Illinois 60510

March 1989

* Presented by J. Dinkel at the 1989 IEEE Particle Accelerator Conference, Chicago, Illinois, March 20-23, 1989. 
John Dinke1, Jawes Biggs

Fermi National Accelerator Laboratory*

P.0. Box 500, Batayia, Illinois 60510

\section{Abstract}

The application of magnetic current amplification and switching techniques to the generation of precise high current pulses for switching magnets is described. The square loop characteristic of Metglas tape wound cores at high excitation levels provides excellent switching characteristics for microsecond pulses. The rugged and passive nature of this type pulser makes it possible to locate the final stages of amplification at the load for maximum efficiency.

\section{Introduction}

With three uniformly spaced beam batches in the Fermilab main ring, extraction for P-bar production may be accomplished with a half sinusoid pulse. A pulsed magnet provides a .45 mad kick to extract the 120 GEV beam. This requires a peak current of $4.2 \mathrm{Ka}$ for extraction at $120 \mathrm{GEV}$ which increases to $5.2 \mathrm{Ka}$ for $150 \mathrm{GEV}$ extraction. The 21 Usec revolution frequency necessitates a 7 Usec batch to batch spacing.

To meet these modest requirements, a pulsed power supply is required which can develop a $6 \mathrm{Ka}$ half sjinusoid current pulse with a base width of 10usec into an inductive load of 8uR. An obvious design is to resonantly discharge a capacitor into the inductive load thru series diodes to prevent current reversal. Unfortunately, the recovery time of the diodes is sufficiently long that the stored energy which accumulates in the inductivo load during diode recovery will break down the diodes when they finally do turn off. The results of this effect as shown in figure 1 indicate that the diodes drop out of conduction 3 Usec after the load current goes through zero. At this time, the load current has reached a level of 1 b00a. As the diodes recover, the reverse voltage generated by Ldi/dt reaches a peak of $16 \mathrm{Kv}$ for this particular case.

The magnetic switch offers an attractive solution to the diode recovery problem and provides the useful side benefit of passive current amplification as rell. This technique has seen widespread use in the area of radar modulators since the early 1950's.1 23 The basic principle behind magnetic switching is to utilize the large changes in impedance which occur when a ferromagnetic material is driven into saturation. By using saturable reactors in the transmission line configuration shown in Figure 2A, this behavior can be used to amplify current in a resonant circuit. Multiple stages are used to achieve additional gain. Consider the case where all capacitors have equal values. When switch $S$ closes, $C_{0}$ is charged thru $L_{0}$ until reactor $L_{1}$ saturates. $C_{0}$ then charges $C_{1}$ thru $L_{1}$ until $L_{2}$ saturates and lets $C_{1}$

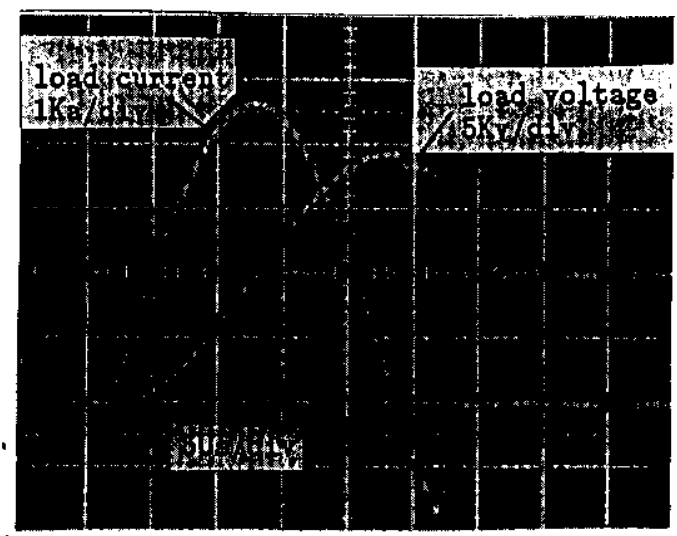

Fig. 1 Effect of diode recovery in a resonant circuit. charge $\mathrm{C}_{2}$ thru $\mathrm{L}_{2}$. The peak current in each of these loops is given by:

$$
I_{p}=V_{c} / Z_{o}=V_{c} \sqrt{C_{n} / 2 L_{n}}
$$

where $V_{C}$ is the peak capacitor voltage. By making the saturated inductance progressively smaller in successive stages, the peak current will increase as the inductance decreases. This is illustrated in Figure 2B.

To examine the circuit in more detail, let us start with Maxwell's equation for time-varying magnetic fields:

$$
\int \mathrm{E} \cdot \mathrm{d} 1=\mathrm{V}_{\mathrm{L}}=\mathrm{N} \mathrm{d} \phi / \mathrm{dt}=\mathrm{NA} \mathrm{dB} / \mathrm{dt}
$$

from which:

$$
\mathrm{V}_{\mathrm{L}} \mathrm{dt}=\mathrm{NA}\left(\mathrm{B}_{\mathrm{g}}-\mathrm{B}_{\mathrm{i}}\right)=\mathrm{NA \Delta B}
$$

where $\mathrm{N}$ is the number of turns around a core having a magnetic cross- sectional area $A, B_{s}$ and $B_{i}$ represent the saturation and initial fields respectively.

The time required to transfer charge from one capacitor to the next is given by:

$$
\mathrm{T}_{\mathrm{t}}=\left(\mathrm{L}_{\mathrm{n}} \mathrm{C} / 2\right) 1 / 2
$$

where $C_{1}=C_{2}=C_{3}=C$. The time required to transfer charge should be approximately equal to the time to

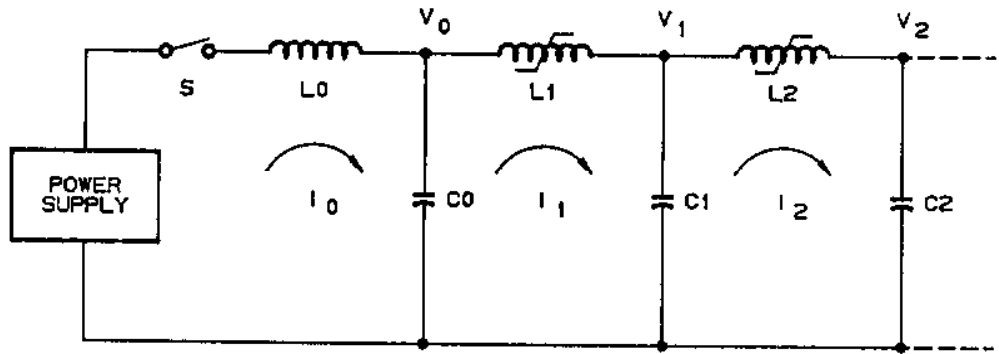

FIGURE 2A

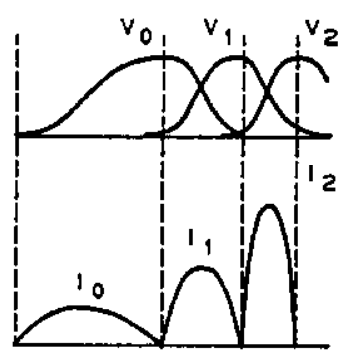

FIGURE 28

* Operated by Universities Research Association, Inc., under contract with the U.S. Department of Energy. 
reach saturation in the core. This leads to the relation:

$$
T_{t}=2\left(B_{S}-B_{i}\right) N A / V_{C}
$$

rhere $V_{C}$ is the capacitor voltage.

The saturable reactor in our design is a coaxial structure for which the inductance is given by:

$$
\mathrm{L}_{\mathrm{S}}=2 \times 10^{-7} 1_{\mathrm{W}} \mathrm{N}^{2} \cdot \ln \left(\mathrm{R}_{\mathrm{o}} / \mathrm{R}_{\mathrm{i}}\right)
$$

where $l_{i p}$ is the reactor length, $R_{0}$ and $R_{i}$ are the outside and inside diameters of the toroidal windings. $Y$ is the number of windings on the reactor.

To perform satisfactorily as a switch, the ratio of the unsaturated inductance to the saturated inductance must be greater than 2000.1 Such a reactor has hysteresis loops similar to those shown in figure 3. Wide flux swings in the core material allows large transfers of energy per pound of core. An abrupt transition between the saturated and unsaturated states produces a correspondingly fast risetime for the switch. Losses and the rounding of the transition into saturation are dependent on $\mathrm{dB} / \mathrm{dt}$ and the propagation of the domain walls in the ferromagnetic tape.45678 510 The net effect of these losses is to produce a magnetizing current during the time that the field is changing from $B_{i}$ to $B_{S}$.

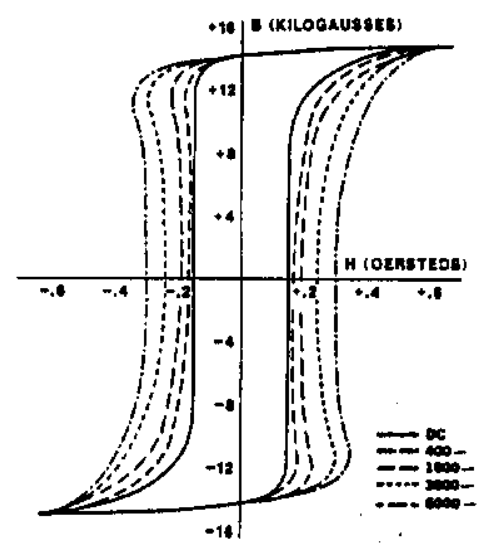

Figure 3 B - B loops for Metglas alloy 2605SC

Toroidal cores for this pulser are made of a .001 inch amorphous alloy 2605SC tape manufactured by Allied Chemical.11 12 They have an I.D. of 7.35 in., an O.D. of $8.96 \mathrm{in.}$, and a height of 2 in. Twelve cores are used in each reactor. Buildup on the cores was purposely kept low since toroidal cores saturate from the inside out due to the difference in magnetic path length. As portions of the core saturate, the effective area is reduced forcing a higher $\mathrm{dB} / \mathrm{dt}$ in the remaining unsaturated areas. This can lead to interlamination sparking. The tape is coated with silicon dioxide to achieve an interlamination breakdown rating of of 2 volts per lamination and a packing factor of $75 \%$. Kapton washers provide insulation between adjacent cores. Bach saturable reactor is contained in a 10 inch diameter aluminum cylinder filled with transformer oil. The windings are tailored around the core stack to provide a uniform current sheet around the cores.

The initial flux density can be set by flowing a RESET current through an auxiliary winding to magnetize the core in the reverse direction. Such a current may be pulsed or DC, but in either case, it must be decoupled from the switching reactor. For this pulser, chokes are used for decoupling. Their effect is to limit load induced current in the RESET circuit to 12 amps.

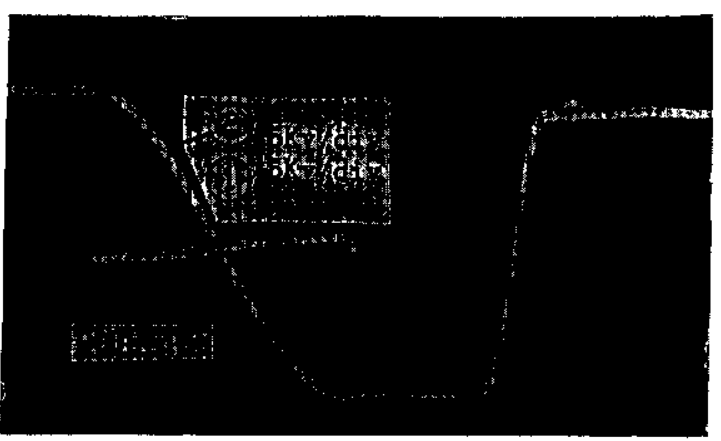

Figure 5

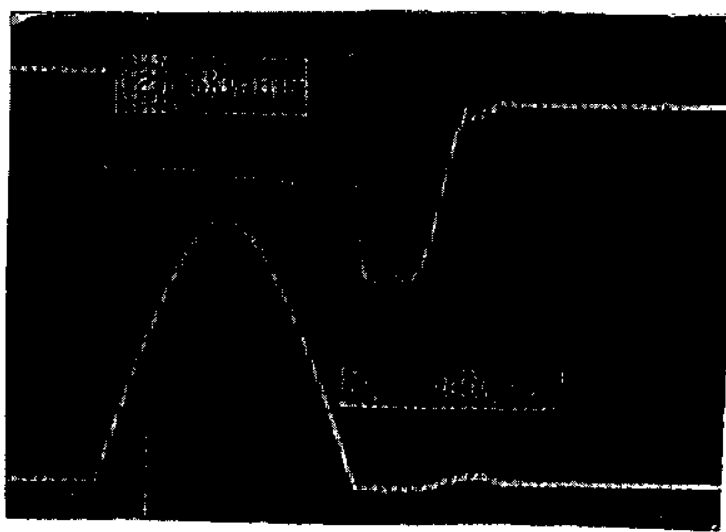

Voltage at node 2 and thyratron anode current Figure 8

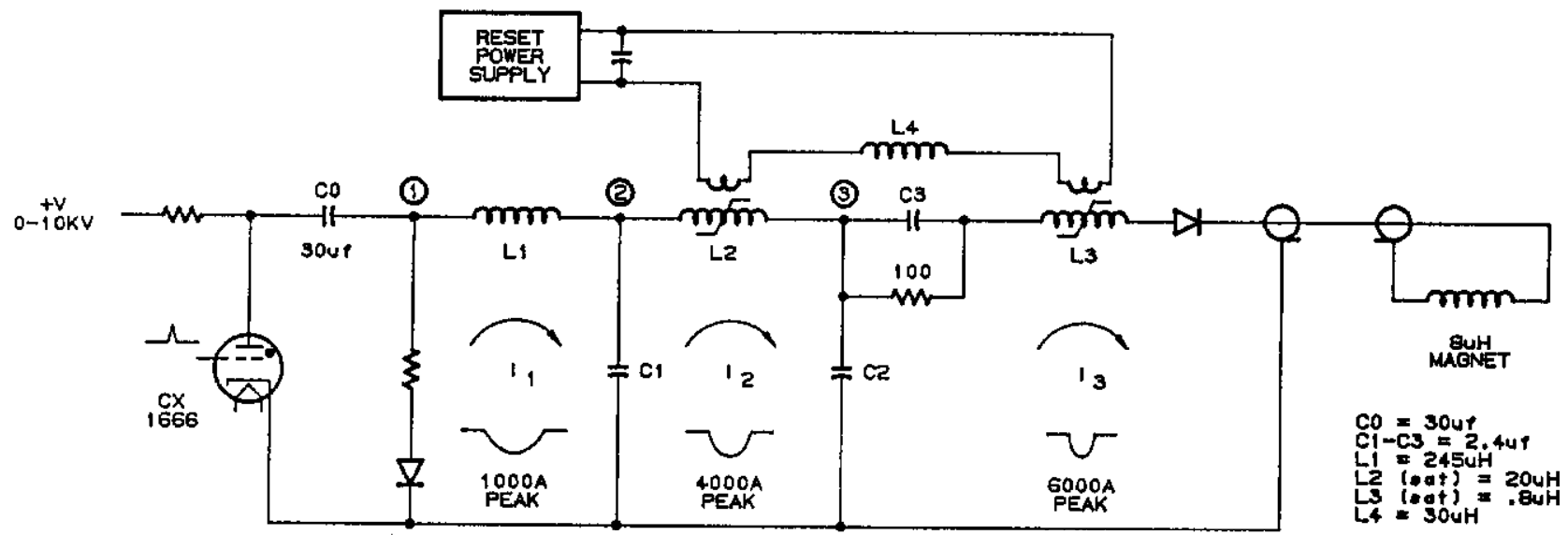

FIGURE 4 MAGNETICALLY SWITCHED PULSER 
The magnetically switched pulser is shown in Pigure 4. $C_{0}$ is charged to a maximum of $10 \mathrm{Kv}$. A hydrogen thyratron switches the voltage on $\mathrm{C}_{0}$ across $\mathrm{L}_{0}$ and $\mathrm{C}_{1}$. Since $\mathrm{C}_{0}$ is much larger than $\mathrm{C}_{1}$, the final. voltage on $C_{1}$ is nearly twice the final voltage on $C_{0}$. Figure 5 shows typical voltages at nodes 1 and 2 of figure 4. when the current in the loop swings through zero as shown in figure 6 , the thyratron turns off and its anode voltage falls to $V_{c 1}$ which has a typical winimum of -19Kv. For a 6Ka output pulse, the peak anode current is approximately 950 amps.

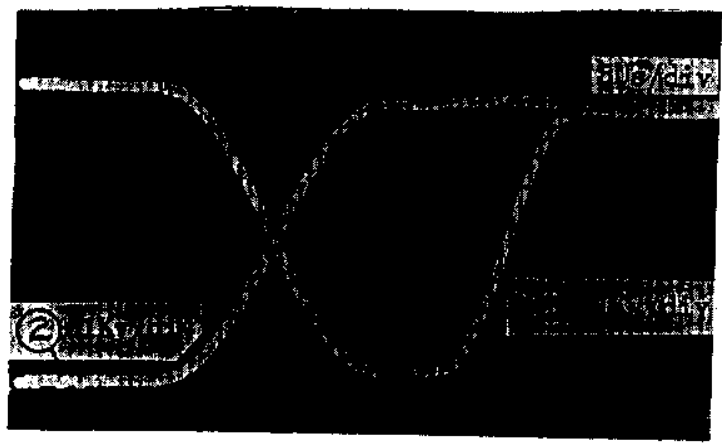

Figure 7

Figure 7 shows the resonant transfer of energy from $\mathrm{C}_{1}$ to $\mathrm{C}_{2}$ after $\mathrm{L}_{2}$ goes into saturation. Figure 8 shows the magnetizing current which occurs during this interval. It appears as a "front porch" on the output current pulse. Following the output pulse, the energy resides as a voltage on $\mathrm{C}_{3}$ which must be dissipated before the next pulse. To prevent this voltage from saturating the switching cores in the reverse direction, a diode stack is placed in series with the load. A "rear porch" follows the output pulse while this diode goes thru the recovery process.

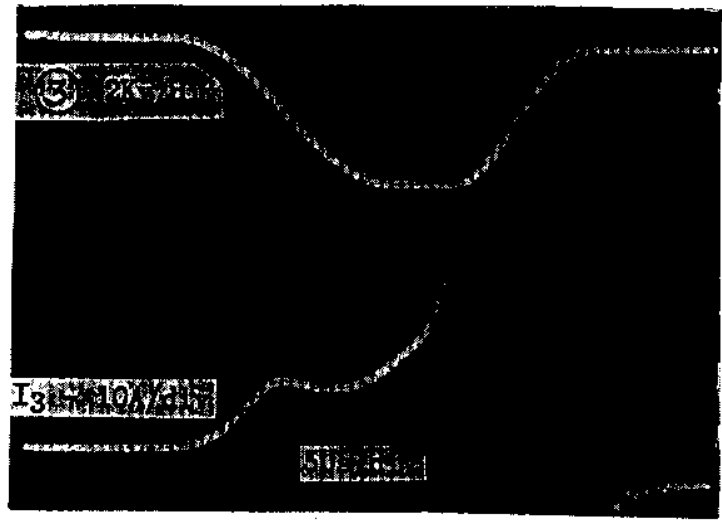

Figure 8

A minimum delay of 85 uSec occurs following a trigger pulse during which time resonant charging (70uSec) and compression (15uSec) take place. This delay is a function of the RESET current which sets the peak to peak flux swing in the saturable reactors. While this delay is slightly temperature sensitive, it is relatively jitter free. This delay is amplitude sensitive, however; which requires a timing adjustment for an amplitude change. Such an adjustment could be eliminated by changing the RESET current or providing a feedback loop which would adjust the RESET current to maintain a fixed propagation delay through the system.
A side benefit of this approach is that amplification is carried out by capacitors and inductors which can be placed in the tunnel environment as part of the pulsed load to minimize power losses in otherwise long high current cable runs. Active elements such as the hydrogen thyratron could then be conveniently located some distance away.

\section{$\underline{\text { References }}$}

1. Melville, W.S.: The Use of Saturable Reactors as Pulse Generators

2. Smith, E.H.: Design and Performance of Magnetic Pulse Modulators

3. Birx, D.L. et al: The Application of Magnetic Switches as Pulse Sources for Induction Linacs

4. Bishop, Jel.: A Comparison of Rapid Surface and Volume Magnetization Measurements on $50 \%$ NiFe Tape With Models of Eddy-current Limited Domain Wall Motion

5. Smith, C.H.: Metallic Glasses in High Energy Pulse Power Systems

6. Jones, R.M.: Setp $\mathrm{dB} / \mathrm{dt}$ Magnetization Losses in Toroidal Amorphous Ribbon and Polycrystalline Cores

7. Swith, C.H.: Fast Magnetization Reversals in Tapepound Square-10op Amorphous Alloy Cores

8. Smith, C.H.: Dynamic Magnetization Of Metallic Glasses

9. Smith, C.H.: Thickness Dependence of Magnetic Losses in Amorphous FeBSiC Ribbon Under Step $\mathrm{dB} / \mathrm{dt}$ Magnetization

10. Smith, C.H.: Hagnetic Losses in Metallic Glasses Under Pulsed Excitation

11. Davis B, L.A.: Metallic Glasses: Formation, Structure, and Properties

12. Nathasingh: A New High Flux Low Loss Magnetic Material for High Frequency Applications 